\title{
Magneto-optics in transition metal diselenide monolayers
}

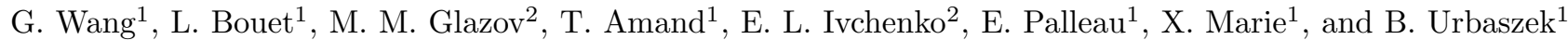 \\ 1 Université de Toulouse, INSA-CNRS-UPS, LPCNO, \\ 135 Avenue de Rangueil, 31077 Toulouse, France and \\ 2 Ioffe Institute, 194021 St.-Petersburg, Russia
}

\begin{abstract}
We perform photoluminescence experiments at $4 \mathrm{~K}$ on two different transition metal diselenide monolayers, namely $\mathrm{MoSe}_{2}$ and $\mathrm{WSe}_{2}$ in magnetic fields $B_{z}$ up to $9 \mathrm{~T}$ applied perpendicular to the sample plane. In $\mathrm{MoSe}_{2}$ monolayers the valley polarization of the neutral and the charged exciton (trion) can be tuned by the magnetic field, independent of the excitation laser polarization. In the investigated $\mathrm{WSe}_{2}$ monolayer sample the evolution of the trion valley polarization depends both on the applied magnetic field and the excitation laser helicity, while the neutral exciton valley polarization depends only on the latter. Remarkably we observe a reversal of the sign of the trion polarization between $\mathrm{WSe}_{2}$ and $\mathrm{MoSe}_{2}$. For both systems we observe a clear Zeeman splitting for the neutral exciton and the trion of about $\pm 2 \mathrm{meV}$ at $B_{z} \mp 9 \mathrm{~T}$. The extracted Landé-factors for both exciton complexes in both materials are $g \approx-4$.
\end{abstract}

\section{INTRODUCTION}

The two-dimensional (2D) semiconductor structures based on monolayer (ML) transition metal dichalcogenides (TMDCs) show very strong light-matter interaction, with an absorption of the order of $10 \%$ per ML in the visible region of the optical spectrum [1-3]. This strong interaction can be explored in microcavity physics and for laser and light emitting diode (LED) devices. The optical properties of ML TMDCs are governed by strongly bound excitons (binding energy is of the order of $0.5 \mathrm{eV}$ ) [4-12]. The absence of an inversion centre in the lattice of TMDC MLs together with the strong spin-orbit (SO) interaction in these materials leads to a coupling of carrier spin and $\boldsymbol{k}$-space valley dynamics. As a result the circular polarization $\left(\sigma^{+}\right.$or $\left.\sigma^{-}\right)$of the absorbed or emitted photon can be directly associated with selective exciton generation in one of the two non-equivalent $K$-valleys: $K^{+}$or $K^{-}$, respectively [13-19]. Excitonic resonances do not only dominate single- and two-photon absorption, but also strongly influence second harmonic generation (SHG) [11] and interactions with plasmons [20]. The exact symmetry, degeneracy and energy spacing of the exciton levels is still under debate. To address the spin- and valley-dependent fine structure of excitonic levels we perform magneto-optical experiments on ML TMDCs, where the magnetic field lifts the underlying degeneracies and extract the effective Lande factors, a technique successfully used in the past to study the bandstructure and excitonic effects of semiconductors [21].

Very recently lifting of the $K$-valley degeneracy through the application of a magnetic field perpendicular to the monolayer plane has been reported for $\mathrm{MoSe}_{2}$ $[22,23]$ and $\mathrm{WSe}_{2}[24,25]$. An energy splitting $\Delta_{Z}$ between the $\sigma^{+}$and $\sigma^{-}$polarized photoluminescence (PL) components has been reported, which correspond to inter-band recombination in the $K^{+}$- and $K^{-}$-valley, respectively [13-17, 26]. The splitting $\Delta_{Z}$ increases roughly linearly with the applied magnetic field, although the values reported for $\mathrm{ML} \mathrm{WSe}_{2}$ differ by a factor of up to $3[24,25]$. The aim of this comparative study is to perform experiments on $\mathrm{MoSe}_{2}$ and $\mathrm{WSe}_{2}$ monolayers in magnetic fields up to $\left|B_{z}\right|=9$ Tesla in the same set-up, and to measure the neutral exciton and trion emission energy and polarization. We vary the laser excitation energy and helicity, which allows us to distinguish between the laser induced and magnetic field induced valley polarization, which show very different dependencies on the applied magnetic field when comparing $\mathrm{MoSe}_{2}$ with $\mathrm{WSe}_{2}$ monolayers.

\section{EXPERIMENTAL SET-UP AND SAMPLES}

$\mathrm{WSe}_{2}$ and $\mathrm{MoSe}_{2}$ flakes are obtained by exfoliation [27] of a bulk crystal on a $\mathrm{SiO}_{2} / \mathrm{Si}$ substrate. The ML regions are identified by optical contrast and very clearly in PL spectroscopy. Experiments at $T=4 \mathrm{~K}$ and in magnetic fields up to $\pm 9 \mathrm{~T}$ have been carried out in an ultra-stable confocal microscope developed for spectroscopy on single semiconductor quantum dots (typical diameter of $20 \mathrm{~nm}$ ) $[28,29]$. We can infer that the mechanical movement of the detection and excitation spot on the sample due to the strong applied magnetic fields is at most in the tens of $\mathrm{nm}$ range in our set-up. The detection spot diameter is about $700 \mathrm{~nm}$. The sample is excited either by a HeNe laser $(1.96 \mathrm{eV})$ or by a tunable continuous wave Ti:Sa laser. The average laser power is in the $\mu \mathrm{W}$ range, in the linear absorption regime. The PL emission is dispersed in a double-monochromator and detected with a Si-CCD camera. The spectral resolution of this detection system is $\approx 20 \mu \mathrm{eV}$. The circular PL polarization $P_{c}$ is defined as

$$
P_{c}=\left(I_{\sigma+}-I_{\sigma-}\right) /\left(I_{\sigma+}+I_{\sigma-}\right),
$$

where $I_{\sigma+}\left(I_{\sigma-}\right)$ denotes the intensity of the right $\left(\sigma^{+}\right)$ and left $\left(\sigma^{-}\right)$circularly polarized emission. Light is $\sigma^{+}$ (right circularly polarized) if electric field vector rotates with time clock-wise provided one looks along the light propagation axis. Similarly the linear PL polarization 
$\mathrm{MoSe}_{2}$ monolayer

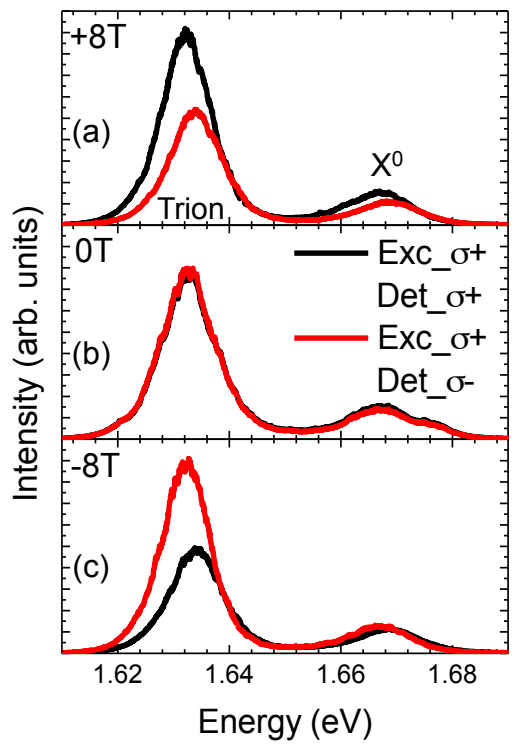

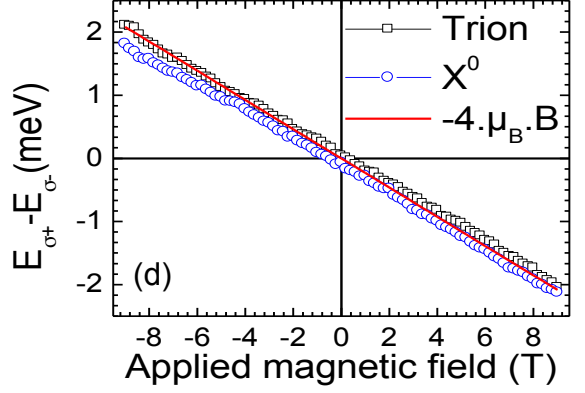

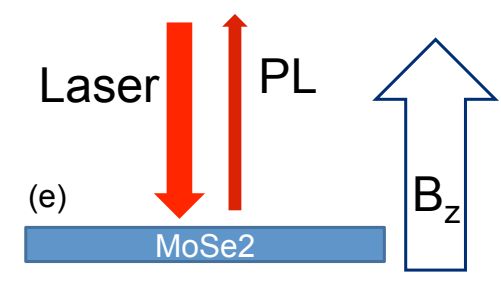

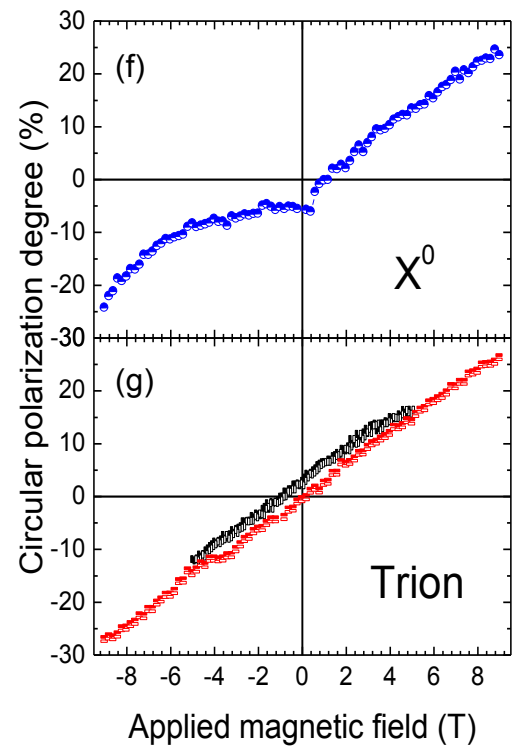

FIG. 1: Data for monolayer MoSe 2 ; excitation energy $E_{\text {Laser }}=1.96 \mathrm{eV}$ (a) PL spectra at $B_{z}=+8 \mathrm{~T}$ for $\sigma^{+}$(black) and $\sigma^{-}$(red) polarized detection using $\sigma^{+}$polarized laser excitation. (b) same as (a) but at $B_{z}=0 \mathrm{~T}$. (c) same as (a) but at $B_{z}=-8 \mathrm{~T}$. (d) Splitting between the $\sigma^{+}$and $\sigma^{-}$polarized PL components for the trion (black squares) and the $\mathrm{X}^{0}(\mathrm{blue}$ circles) as a function of magnetic field, the function $-4 \mu_{B} B_{z}$ is shown for comparison (red line). (e) Schematics of the experimental geometry. (f) Polarization of the PL emission of the $\mathrm{X}^{0}$ as a function of $B_{z}$. (g) Polarization $P_{c}$ of the PL emission of the trion as a function of $B_{z}$ using $\sigma^{+}$(black squares) and $\sigma^{-}$excitation (red squares).

in the fixed (xy) axes lying in the sample plane writes $P_{l}=\left(I_{x}-I_{y}\right) /\left(I_{x}+I_{y}\right)$ with $I_{x}\left(I_{y}\right)$ the $x$ and $y$ linearly polarized emission components.

\section{MAGNETO-OPTICS IN MONOLAYER $\mathrm{MoSe}_{2}$}

First we discuss the experimental results for $\mathrm{MoSe}_{2}$ MLs. At zero magnetic field, we observe in the PL spectrum two sharp emission features with a full width at half maximum (FWHM) of $10 \mathrm{meV}$. In accordance with previous reports [6], the low energy emission at $1.63 \mathrm{eV}$ is attributed to the charged exciton (trion) recombination and at $1.67 \mathrm{eV}$ we record the neutral exciton $\mathrm{X}^{0}$ emission, see Fig. 1b. In our measurements the circular polarization degree $P_{c}$ of the trion and $\mathrm{X}^{0}$ emission depends only very little on the excitation laser polarization $\left(\sigma^{+}\right.$or $\left.\sigma^{-}\right)$for the excitation laser energy of $1.96 \mathrm{eV}$ used in Fig. 1b. The maximum $P_{c}$ generated was of the order of $5 \%$ for the excitation energy range investigated [30], much lower than the PL polarization achieved for circular laser excitation in $\mathrm{MoS}_{2}[14-17,26]$ and $\mathrm{WSe}_{2}$ MLs $[11,18,31]$. Below we discuss the magnetic field dependence of the PL emission, whose energy and polarization are essentially independent of the excitation laser polarization, in stark contrast to the results obtained in $\mathrm{ML}$ $\mathrm{WSe}_{2}$ discussed in Sec. IV.

Next we discuss the changes observed in the PL emis- sion when applying a magnetic field $B_{z}$ perpendicular to the layer plane, i.e. along the $z$-direction, that is also the light propagation axis (Faraday geometry). Comparing Fig. $1 \mathrm{a}$ at $B_{z}=+8 \mathrm{~T}$ and Fig. $1 \mathrm{~b}$ at $B_{z}=0$ we observe two main differences: (i) For $B_{z}=+8 T$ the $\sigma^{+}$ polarized emission is more intense than the $\sigma^{-}$polarized component, for both the trion and $\mathrm{X}^{0}$; (ii) The $\sigma^{+}$polarized emission is shifted to lower energy compared to the $\sigma^{-}$polarized component. When applying a field of $B_{z}=-8 \mathrm{~T}$ in Fig. 1c, these results are reversed, i.e. $\sigma^{-}$ emission becomes more intense and lower in energy compared to $\sigma^{+}$in agreement with time-reversal symmetry. In Fig. 1d we plot the full magnetic field dependence of the energy splitting

$$
\Delta_{Z}=E_{\sigma^{+}}^{P L}-E_{\sigma^{-}}^{P L}=g \mu_{B} B_{z}
$$

from $B_{z}=-9 \mathrm{~T}$ to $+9 \mathrm{~T}$ with $g$ being the effective $g$ factor and $\mu_{B}$ being the Bohr magneton. The Zeeman splitting is extracted by fitting the trion and $\mathrm{X}^{0}$ emission spectra with Lorentzians. We observe a clear linear dependence both for the trion and $\mathrm{X}^{0}$ splitting on the applied field. This indicates that eventual diamagnetic and higher order contributions are identical for the upper and lower Zeeman branch within our experimental resolution. The slope is $\Delta_{Z} / B_{z}=-220 \pm 10 \mu \mathrm{eV} / \mathrm{T}$ for the $\mathrm{X}^{0}$ which corresponds to an exciton $g$-factor of $g_{X^{0}}=-3.8 \pm 0.2$. For the trion the slope is $-226 \pm 10 \mu \mathrm{eV} / \mathrm{T}$ which corresponds to a $g$-factor of $g_{T}=-3.9 \pm 0.2$. This corresponds to a maximum $\Delta_{Z}=-2 \mathrm{meV}$ at 9 Tesla. The 
main experimental uncertainty for $\Delta_{Z}$ comes from eventual changes of the overall shape of the PL emission due to imperfections in the optical set-up and sample inhomogeneities, as the recorded shifts are smaller than the linewidth. The Landé-factors for trions and the $\mathrm{X}^{0}$ in ML $\mathrm{MoSe}_{2}$ extracted from our date are close to the values reported in [22, 23].

It is worth mentioning that trions that form with the excess electron in the same or different valley, with respect to the photo-generated electron hole pair, are separated in energy due to the strong Coulomb effects and the zero-field splitting of conduction band states in each valley, cf. [32]. However, we do not observe any fine structure splitting for the trion emission in the investigated sample. Hence, the measured $g_{T}$ represents the global magnetic field induced energy shift of the trion emission.

We now discuss the circular polarization of the observed emission summarized in Figs. 1f and 1g. For the trion, $P_{c}$ increases from zero to $30 \%$ when the magnetic field is increased from zero up to $9 \mathrm{~T}$. This strong polarization is reversed when the direction of the applied magnetic field is reversed. Optical valley initialization at higher fields is not at the origin of this effect: For $\sigma^{+}$ polarized laser excitation we observed exactly the same polarization increase with field as for $\sigma^{-}$polarized laser excitation. We conclude that the PL polarization is the result of magneto-induced spin/valley polarization buildup during the PL emission time, which has been determined to be in the ps-range [30]. Observing an increase in the emission polarization in applied magnetic fields is a very common observation in semiconductors [33]. For $\mathrm{ML} \mathrm{MoSe}$, where excitons have a strong binding energy [8], this observation is very surprising taking into account the relevant energy scales. At 9 Tesla, we have induced a splitting between the valley Zeeman levels of $2 \mathrm{meV}$, eventually comparable to the spin splitting in the conduction band, predicted to be in the meV range [34, 35]. The polarization of the neutral exciton follows a similar trend as the trion. For the neutral exciton, the applied field $B_{z}$ can dominate the long-range electron-hole Coulomb exchange interaction, and hence suppress valley depolarization when $B_{z}$ has a larger amplitude than the effective transverse field associated to exchange effects [32, 36-38].

\section{MAGNETO-OPTICS IN MONOLAYER WSe}

In order to draw more general conclusions on valley properties in applied magnetic fields, we compare our results in ML MoSe 2 with a very well characterized system, namely $\mathrm{ML} \mathrm{WSe}_{2}[11,18,31]$ investigated with the same experimental set-up. Therefore all experimental uncertainties are comparable. In Fig. $2 b$ the PL emission of $\mathrm{ML} \mathrm{WSe} 2$ is plotted, and just as in the case of ML MoSe $\mathrm{ML}_{2}$ we observe two well defined emission lines with a typical FWHM of $10 \mathrm{meV}$. The higher energy transition at $1.75 \mathrm{eV}$ is identified as the neutral exciton $\mathrm{X}^{0}$ recombination, as valley coherence can be generated [18], see
TABLE I: comparison of Landé factors

\begin{tabular}{ccccc}
\hline \hline$g_{X^{0}}$ & $\begin{array}{c}\mathrm{X}^{0} \text { slope } \\
{[\mu \mathrm{eV} / \mathrm{T}]}\end{array}$ & $g_{T}$ & $\begin{array}{c}\text { Trion slope } \\
{[\mu \mathrm{eV} / \mathrm{T}]}\end{array}$ \\
\hline $\mathrm{ML} \mathrm{MoSe}_{2}$ & $-3.8 \pm 0.2$ & $-220 \pm 10$ & $-3.9 \pm 0.2$ & $-226 \pm 10$ \\
$\mathrm{ML} \mathrm{WSe}_{2}$ & $-3.7 \pm 0.2$ & $-214 \pm 10$ & $-4.4 \pm 0.2$ & $-254 \pm 10$ \\
\hline \hline
\end{tabular}

Fig. 2e. The lower energy transition at $1.72 \mathrm{eV}$ stems from the radiative recombination of the charged exciton (trion). The $\sigma^{+}$and $\sigma^{-}$polarized PL components correspond to carrier recombination in the $K^{+}$and $K^{-}$valley, respectively. At zero magnetic field the emission spectra in $\sigma^{+}$and $\sigma^{-}$polarizations are exactly the same due to the time-reversal symmetry and corresponding degeneracy of the valley levels. In a magnetic field of $9 \mathrm{~T}$ this valley degeneracy is lifted, and the energy difference $\Delta_{Z}=E_{\sigma^{+}}^{P L}-E_{\sigma^{-}}^{P L}$, Eq. (2), is typically $-2 \mathrm{meV}$, see Fig. 2a, very similar to the results on $\mathrm{MoSe}_{2}$ presented in Fig. 1a. In a field of $B_{z}=-9 \mathrm{~T}$, the $\sigma^{+}$polarized component is now at higher energy, corresponding to a positive Zeeman splitting of $+2 \mathrm{meV}$. We plot in Fig. $2 \mathrm{~d}$ the full magnetic field dependence of the Zeeman splitting for the trion and $\mathrm{X}^{0}$. The experimental points are very close to a straight line, which allows us to extract for the $\mathrm{X}^{0}$ a slope of $\Delta_{Z} / B_{z}=-214 \pm 10 \mu \mathrm{eV} / \mathrm{T}$, corresponding to the neutral exciton $g$-factor $g_{X^{0}}=-3.7 \pm 0.2$. For the trion the slope is $-254 \pm 10 \mu \mathrm{eV} / \mathrm{T}$, resulting in its $g$-factor $g_{T}=-4.4 \pm 0.2$.

The similarities between the measured Landé-factors in $\mathrm{MoSe}_{2}$ and $\mathrm{WSe}_{2}$ are striking, both with respect to their signs and amplitudes, see table I. Comparing with the very recent literature data on $\mathrm{ML} \mathrm{WSe}_{2}$, the $g$ factors for the neutral exciton extracted for our sample are smaller than reported in reference [25] and larger than in reference [24]. Note, that as for $\mathrm{MoSe}_{2}$ case we do not resolve any fine-structure of the trion emission in $\mathrm{WSe}_{2}$.

Although the Zeeman energy evolution of the both ML materials $\mathrm{MoSe}_{2}$ and $\mathrm{WSe}_{2}$ shows close similarities, we will see below that the evolution of the valley polarization as a function of the applied magnetic field is completely different. The polarization measurements on $\mathrm{WSe}_{2}$ are all performed with non-resonant HeNe laser excitation. First, we plot the evolution of the $\mathrm{X}^{0} \mathrm{PL}$ polarization under polarized pumping, that corresponds to the valley polarization generation via the chiral optical selection rules $[13,14]$, as a function of the applied magnetic field. It can be seen in Fig. $2 \mathrm{f}$ that the $\mathrm{X}^{0}$ polarization is essentially independent of the applied field. For a linearly polarized excitation laser, we find $P_{c} \approx 0$ for all applied field values. Changing to $\sigma^{+}$circularly polarized excitation, we record at zero Tesla $P_{c} \approx 40 \%$. This value remains practically constant in applied fields from $-9 \mathrm{~T}$ to $+9 \mathrm{~T}$. This indicates that over the entire magnetic field range, the $\mathrm{X}^{0}$ emission polarization is determined by the initially, optically created valley polarization rather than induced by the magnetic field. During the $\mathrm{X}^{0} \mathrm{PL}$ emission time no valley/spin relaxation occurs, which might 
$\mathrm{WSe}_{2}$ monolayer

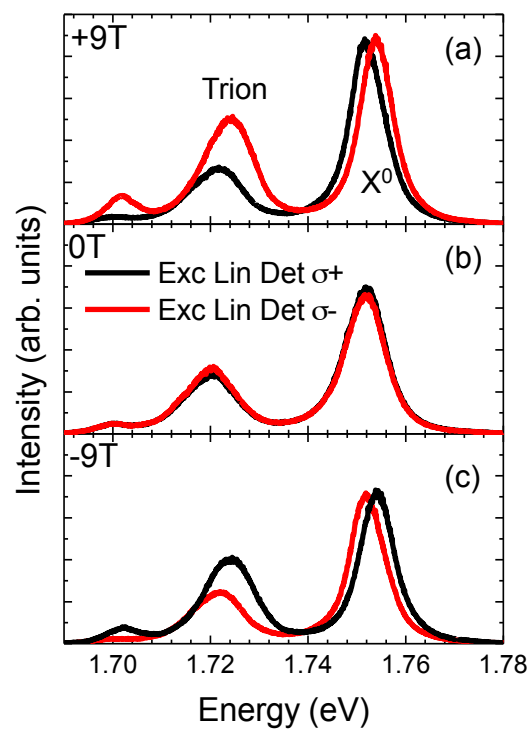

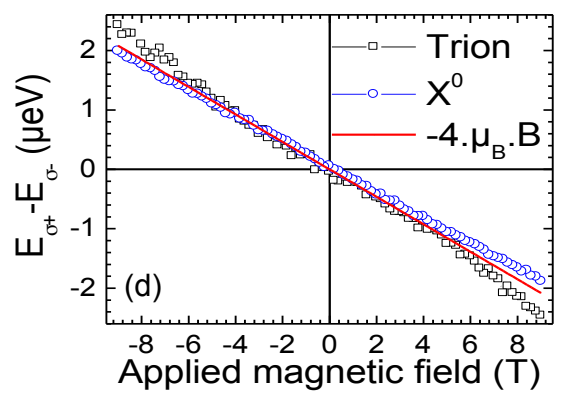

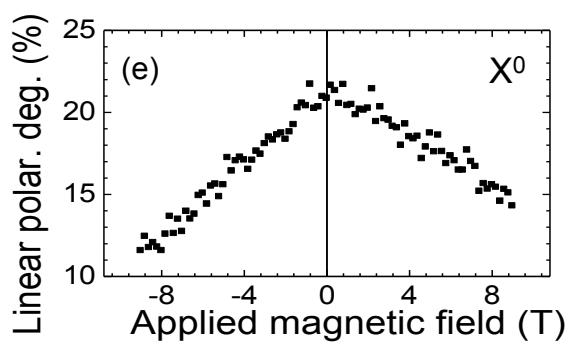

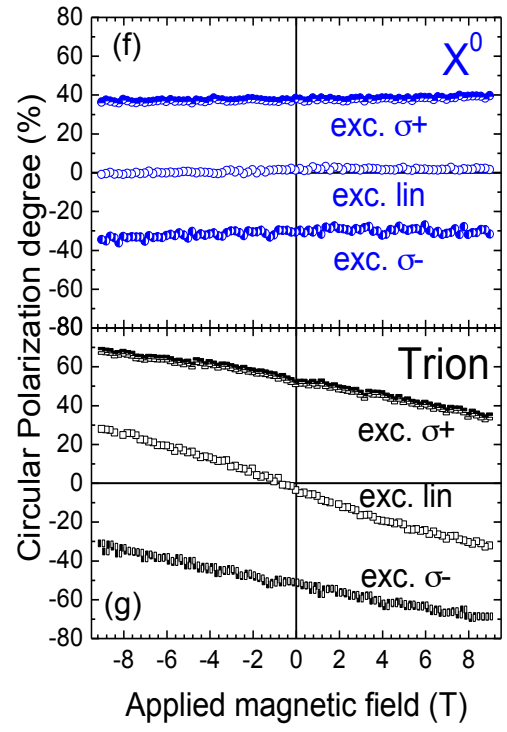

FIG. 2: Data for monolayer WSe $\mathbf{W}_{2}$; excitation energy $E_{\text {Laser }}=1.96 \mathrm{eV}$ (a) PL spectra at $B_{z}=+9 \mathrm{~T}$ for $\sigma^{+}(\mathrm{black})$ and $\sigma^{-}$(red) polarized detection using $\sigma^{+}$polarized laser excitation. (b) same as (a) but at $B_{z}=0 \mathrm{~T}$. (c) same as (a) but at $B_{z}=-9 \mathrm{~T}$. (d) Splitting between the $\sigma^{+}$and $\sigma^{-}$polarized PL components for the trion (black squares) and the $\mathrm{X}^{0}$ (blue circles) as a function of magnetic field, the function $-4 \mu_{B} B_{z}$ is shown for comparison (red line). (e) Linear polarization of the $\mathrm{X}^{0}$ emission as a function of $B_{z}$. (f) Polarization of the PL emission of the $\mathrm{X}^{0}$ as a function of $B_{z}$ for three different laser polarizations. (g) Polarization $P_{c}$ of the PL emission of the trion as a function of $B_{z}$ for three different laser polarizations.

simply be a consequence of the extremely short PL emission time in the few ps range measured for this material [31]. Exciting preferentially the $K^{-}$valley with a $\sigma^{-}$polarized laser, results in $P_{c} \approx-40 \%$, again independent of the applied magnetic field. This behaviour for the $\mathrm{X}^{0}$ in $\mathrm{ML} \mathrm{WSe}_{2}$ is in stark contrast to the observations in ML $\mathrm{MoSe}_{2}$. For the former, the optical valley initialization determines the PL polarization, for the latter the applied magnetic field direction and amplitude allow to control the valley polarization. Comparing with the recent literature, the $\mathrm{X}^{0}$ polarization in the $\mathrm{ML} \mathrm{WSe}_{2}$ sample investigated by Aivazian et al. [24] was slightly more sensitive to the applied magnetic field.

Contrary to the $\mathrm{X}^{0}$ in our $\mathrm{ML} \mathrm{WSe}_{2}$ sample, the trion polarization in this ML can be controlled via the applied magnetic field, shown in Fig. 2g. For linearly polarized laser excitation, the trion PL polarization for $B_{z}=0$ is absent, $P_{c}=0$, but increases at $-9 \mathrm{~T}$ to $+30 \%$, and at $+9 \mathrm{~T}$ to $-30 \%$. This can be directly compared to the trion in ML $\mathrm{MoSe}_{2}$, that also started off at zero field with zero polarization. When comparing the trion polarization evolution in $\mathrm{ML} \mathrm{MoSe}_{2}$ (Fig. 1g) and ML WSe (Fig. 2g) we note very contrasting behaviour: Application of a positive magnetic field, results in a strong, positive $P_{c}$ in $\mathrm{MoSe}_{2}$ in contrast to the strong, but negative polarization created in $\mathrm{WSe}_{2}$. Using $\sigma^{+}$polarized excitation for the trion, results in high circular polarization $P_{c}=50 \%$ already at $B_{z}=0$. At a magnetic field of $B_{z}=-9 \mathrm{~T}$ this polarization is increased to $P_{c}=68 \%$, at $B_{z}=+9 \mathrm{~T}$ we find $P_{c}=34 \%$. For the trion using $\sigma^{+}$excitation, we find a similar trend as under linearly polarized excitation: the PL polarization decreases for positive $B_{z}$ and increases for negative $B_{z}$. The trion polarization depends on both the excitation laser polarization and the applied magnetic field. This latter dependence might be linked to the fact that the PL emission time of the trion in $\mathrm{ML} \mathrm{WSe}_{2}$ is longer than for the $\mathrm{X}^{0}$ [31], allowing for polarization relaxation to occur before radiative recombination takes place. As can be seen in Fig. 2g, the magnetic field induced change in PL polarization is also observed for $\sigma^{-}$excitation, here we find at $B_{z}=-9 \mathrm{~T} P_{c}=-31 \%$, while for zero field we record $P_{c}=-52 \%$ (as expected when switching from $\sigma^{+}$to $\sigma^{-}$excitation) evolving towards $P_{c}=-69 \%$ for $B_{z}=-9 \mathrm{~T}$. The three different measurement series presented in Fig. $2 \mathrm{~g}$ each confirm that the PL polarization of the trion in $\mathrm{ML} \mathrm{WSe}_{2}$ strongly decreases with the applied magnetic field, whereas, in stark contrast, the trion $\mathrm{PL}$ polarization in $\mathrm{ML} \mathrm{MoSe}_{2}$ strongly increases as a function of the applied $B_{z}$.

\section{DISCUSSION}

The emission energies and polarizations are determined by several factors. Most importantly, the Zeeman splittings of exciton and trion in the emission spectra are governed by an interplay of spin splittings of conduc- 
tion and valence band states, while the polarization is governed by (i) selection rules at optical transitions, (ii) occupancies of the spin/valley states and possible spin relaxation processes. Here we briefly discuss theoretical approaches to evaluate Zeeman splittings and polarization of emitted radiation in the context of experimental data presented above.

\section{A. Zeeman effect in two-dimensional crystals}

We recall that the electron wavefunction in the twodimensional crystal can be recast, in accordance with the Bloch theorem, as

$$
\psi_{n \boldsymbol{q}}(\boldsymbol{r})=\frac{\mathrm{e}^{\mathrm{i} \boldsymbol{q} \boldsymbol{r}}}{\sqrt{S}} u_{n \boldsymbol{q}}(\boldsymbol{r}),
$$

where $n$ enumerates bands (including electron spin state) and $\boldsymbol{q}$ is the quasi-wavevector, $u_{n \boldsymbol{q}}(\boldsymbol{r})$ is the periodic amplitude normalized per volume of the unit cell, $\Omega_{0}$ : $\int_{\Omega_{0}}\left|u_{n \boldsymbol{q}}\right|^{2} d \boldsymbol{r}=\Omega_{0}$, and $S$ is the macroscopic normalization area.

The bare electron Zeeman effect is described by

$$
\mathcal{H}_{0}=g_{0} \mu_{B} \boldsymbol{B} \cdot \boldsymbol{s}
$$

where $\mu_{B}=|e| \hbar /\left(2 m_{0} c\right)$ is the Bohr magneton, $m_{0}$ and $g_{0}=2$ are the free electron mass and Landé factor, and $\boldsymbol{s}$ is the spin operator $\boldsymbol{\sigma} / 2, \boldsymbol{\sigma}$ being the vector composed of the Pauli matrices. In crystals, in addition to (4) the orbital contribution to the Zeeman splitting should be taken into account. It is related to the orbital momentum of the electron [39],

$$
\mathcal{H}_{1}=\mu_{B} \boldsymbol{B} \cdot \boldsymbol{L}
$$

where $\boldsymbol{L}=\hbar^{-1}[\boldsymbol{r} \times \boldsymbol{p}]$ is the angular momentum operator, and $\boldsymbol{p}=-\mathrm{i} \hbar \boldsymbol{\nabla}$ is the electron momentum operator. The contribution (5) is known to be important for well characterized semiconductors such as GaAs [21, 40].

We recall that in $\mathrm{MoSe}_{2}$ and $\mathrm{WSe}_{2}$ MLs the direct band gaps are realized at the edges of the Brillouin zone characterized by the wavevectors $\boldsymbol{K}^{ \pm}$. The bands at $\boldsymbol{q}=\boldsymbol{K}^{ \pm}$ are non-degenerate and can be characterized by a certain spin projection $s_{z}= \pm 1 / 2$ onto the sample normal. The time reversal symmetry couples $s_{z}= \pm 1 / 2$ states in the $\boldsymbol{K}^{+}$valley with $s_{z}=\mp 1 / 2$ states in the $\boldsymbol{K}^{-}$valley. In what follows we will be interested in the Zeeman effect in magnetic field $\boldsymbol{B} \| z$. Correspondingly, it is instructive to present the diagonal matrix element of $L_{z}$ operator at $\boldsymbol{q}=\boldsymbol{K}^{+}$or $\boldsymbol{K}^{-}$as

$$
\begin{aligned}
& \left\langle\psi_{n}\left|\hbar L_{z}\right| \psi_{n}\right\rangle_{ \pm}= \\
& \sum_{m \neq n}\left[\Omega_{n m}^{x}\left(\boldsymbol{K}^{ \pm}\right) p_{m n}^{y}\left(\boldsymbol{K}^{ \pm}\right)-\Omega_{n m}^{y}\left(\boldsymbol{K}^{ \pm}\right) p_{m n}^{x}\left(\boldsymbol{K}^{ \pm}\right)\right]
\end{aligned}
$$

Here $p_{n m}^{\alpha}(\alpha=x, y)$ are the matrix elements of the electron momentum and $\Omega_{n m}^{\alpha}=\mathrm{i} \Omega_{0}^{-1} \int_{\Omega_{0}} u_{n}^{*}\left(\partial u_{m} / \partial q_{\alpha}\right) d \boldsymbol{r}$ are the interband matrix elements of the coordinate operator. In derivation of Eq. (6) we made use of the completeness relation for the Bloch amplitudes and took into account that $p_{n n}\left(\boldsymbol{K}^{ \pm}\right)=0$. Taking into account that $\Omega_{m n}^{\alpha}=\mathrm{i} \hbar p_{m n}^{\alpha} /\left[\left(E_{n}-E_{m}\right) m_{0}\right]$ with $E_{n}, E_{m}$ being the energies of corresponding bands, Eq. (6) can be rewritten as [40]

$$
\begin{aligned}
& \left\langle\psi_{n}\left|L_{z}\right| \psi_{n}\right\rangle_{ \pm}= \\
& \frac{\mathrm{i}}{m_{0}} \sum_{m \neq n} \frac{p_{n m}^{x}\left(\boldsymbol{K}^{ \pm}\right) p_{m n}^{y}\left(\boldsymbol{K}^{ \pm}\right)-p_{n m}^{y}\left(\boldsymbol{K}^{ \pm}\right) p_{m n}^{x}\left(\boldsymbol{K}^{ \pm}\right)}{E_{m}-E_{n}}
\end{aligned}
$$

Equations (4), (5) and (7) can be used to evaluate Zeeman splittings both in the $\boldsymbol{k} \cdot \boldsymbol{p}$ and tight-binding models, as detailed in the next two subsections.

\section{B. kp-theory}

The multiband $\boldsymbol{k} \cdot \boldsymbol{p}$ model was formulated for TMDCs in Refs. [41-43]. The effective Hamiltonians describing the states in the vicinity of $\boldsymbol{K}^{ \pm}$edges of the Brillouin zone have the form

$$
\begin{gathered}
\mathcal{H}_{+}=\left(\begin{array}{cccc}
E_{c+2} & \gamma_{6} k_{-} & \gamma_{4} k_{+} & 0 \\
\gamma_{6} k_{+} & E_{c} & \gamma_{3} k_{-} & \gamma_{5} k_{+} \\
\gamma_{4} k_{-} & \gamma_{3} k_{+} & E_{v} & \gamma_{2} k_{-} \\
0 & \gamma_{5} k_{-} & \gamma_{2} k_{+} & E_{v-3}
\end{array}\right), \\
\mathcal{H}_{-}=\left(\begin{array}{cccc}
E_{c+2} & \gamma_{6} k_{+} & \gamma_{4} k_{-} & 0 \\
\gamma_{6} k_{-} & E_{c} & \gamma_{3} k_{+} & \gamma_{5} k_{-} \\
\gamma_{4} k_{+} & \gamma_{3} k_{-} & E_{v} & \gamma_{2} k_{+} \\
0 & \gamma_{5} k_{+} & \gamma_{2} k_{-} & E_{v-3}
\end{array}\right) .
\end{gathered}
$$

Here $k_{ \pm}=k_{x} \pm \mathrm{i} k_{y}$ are the cyclic components of the electron wavevector reckoned from the $\boldsymbol{K}^{ \pm}$points, $\boldsymbol{k}_{ \pm}=$ $\boldsymbol{K}^{ \pm}-\boldsymbol{q}$; the parameters $\gamma_{3} \ldots \gamma_{6}$ are related to the interband momentum matrix elements, and certain convention about the phases of the Bloch functions is assumed. The symbols $c+2$ and $v-3$ denote excited conduction and deep valence bands. Such a model was shown to allow for adaquate description of the spin-orbit coupling and trigonal symmetry effects in TMDCs [41-43]. In the general theory besides $\boldsymbol{k}$-linear off-diagonal terms, effective $\boldsymbol{k} \cdot \boldsymbol{p}$ Hamiltonian includes diagonal quadratic in $\boldsymbol{k}$ contributions resulting (i) from the second-order $\boldsymbol{k} \cdot \boldsymbol{p}$ coupling with distant bands and (ii) from bare electron dispersion $\hbar^{2} k^{2} / 2 m_{0}$ [44]. Usually these contributions are comparable and should be included simultaneously. Diagonalizing Hamiltonians (8) in the second order in offdiagonal terms we obtain for the electron effective masses of the conduction and valence bands, respectively,

$$
\begin{aligned}
\frac{1}{m_{c}} & =\frac{1}{m^{*}}+\frac{1}{m_{c}^{\prime}}+\frac{1}{m_{0}}+\frac{1}{m_{c}^{\prime \prime}}, \\
\frac{1}{m_{v}} & =-\frac{1}{m^{*}}+\frac{1}{m_{v}^{\prime}}+\frac{1}{m_{0}}+\frac{1}{m_{v}^{\prime \prime}} .
\end{aligned}
$$


Here subscripts $c, v$ denote the corresponding bands and we use the electron representation, $m^{*}=\hbar^{2}\left(E_{c}-\right.$ $\left.E_{v}\right) /\left(2 \gamma_{3}^{2}\right)$ is the two-band effective mass, the terms

$$
\begin{aligned}
& \frac{1}{m_{c}^{\prime}}=\frac{2}{\hbar^{2}}\left(\frac{\gamma_{5}^{2}}{E_{c}-E_{v-3}}+\frac{\gamma_{6}^{2}}{E_{c}-E_{c+2}}\right), \\
& \frac{1}{m_{v}^{\prime}}=\frac{2}{\hbar^{2}}\left(\frac{\gamma_{2}^{2}}{E_{v}-E_{v-3}}+\frac{\gamma_{4}^{2}}{E_{v}-E_{c+2}}\right)
\end{aligned}
$$

result from the mixing described by the Hamiltonians (8) and $1 / m_{c}^{\prime \prime}, 1 / m_{v}^{\prime \prime}$ contain above mentioned contributions from remote bands [not included in Eqs. (8)].

According to the general theory [44] the magnetic field within the $\boldsymbol{k} \cdot \boldsymbol{p}$ scheme is included (i) by adding the bare Zeeman effect in the form of Eq. (4) and (ii) by replacing $\boldsymbol{k}$ in Eqs. (8) by $\boldsymbol{k}-(e / c \hbar) \boldsymbol{A}$, where $e=-|e|$ is the electron charge, $\boldsymbol{A}$ is the vector potential of the magnetic field. The calculation in the first order in $B_{z}$ yields the effective $g$-factors of electrons in $\boldsymbol{K}^{ \pm}$valleys:

$$
g_{c, v}^{\boldsymbol{K}^{+}} \equiv g_{c, v}=2+g_{c, v}^{\text {orb }}, \quad g_{c, v}^{\boldsymbol{K}^{-}}=-g_{c, v}^{\boldsymbol{K}^{+}} .
$$

The term 2 in Eq. (11) arises from the bare Zeeman effect, Eq. (4), while $g_{c, v}^{\text {orb }}$ result from the $\boldsymbol{k} \cdot \boldsymbol{p}$-mixing

$$
\begin{gathered}
g_{c}^{\mathrm{orb}}=\frac{4 m_{0}}{\hbar^{2}}\left(\frac{\gamma_{3}^{2}}{E_{c}-E_{v}}-\frac{\gamma_{5}^{2}}{E_{c}-E_{v-3}}-\frac{\gamma_{6}^{2}}{E_{c}-E_{c+2}}\right), \\
g_{v}^{\text {orb }}=\frac{4 m_{0}}{\hbar^{2}}\left(-\frac{\gamma_{3}^{2}}{E_{v}-E_{c}}+\frac{\gamma_{2}^{2}}{E_{v}-E_{v-3}}+\frac{\gamma_{4}^{2}}{E_{v}-E_{c+2}}\right) .
\end{gathered}
$$

Equations (12) can be derived from Eqs. (5) and (7), taking into account only bands $c, c+2, v$ and $v-3$. Additional contribution to $g_{c, v}^{\text {orb }}$, namely, $\Delta g_{c, v}^{\text {orb }}$ may arise allowing for other distant bands in Eq. (7).

\section{Tight-binding theory}

In the tight-binding approximation the Bloch function in Eq. (3) is presented as a linear combination of atomic orbitals $\phi_{j}^{a}(\boldsymbol{r})$ (generally orthogonalized [45]) in the form

$$
\psi_{n \boldsymbol{q}}(\boldsymbol{r})=\sum_{a, l, j} \mathrm{e}^{\mathrm{i} \boldsymbol{q} \boldsymbol{R}_{a, l}} C_{l, j}^{a} \phi_{j}^{a}\left(\boldsymbol{r}-\boldsymbol{R}_{a, l}\right) .
$$

Here $a$ enumerates types of atoms ( $a=$ Metal or Chalcogen), $l$ enumerates atoms of a given type, $j$ runs through the set of orbitals taken into account at a given atom, $\boldsymbol{R}_{a, l}$ are the positions of the atoms in the two-dimensional lattice and $C_{l, j}^{a} \equiv C_{l, j}^{a}(n, \boldsymbol{q})$ are the coefficients. The tight-binding Hamiltonian acting in the space of coefficients $C_{j, l}^{a}$ contains diagonal energies, i.e. energies of orbitals, and hopping matrix elements mixing coefficients $C_{l, j}^{a}$ and $C_{l^{\prime}, j^{\prime}}^{a^{\prime}}$ for different atoms $\left(l \neq l^{\prime}\right)$. There are several tight-binding models developed for TMDC monolayers [46]: (i) the 3-band model [35] which takes into account only three $d$-orbitals of Metal, namely, $d_{z^{2}}, d_{x^{2}-y^{2}}$, and $d_{x y}$, and includes up to three nearest neighbours to reproduce density functional theory (DFT) calculations of electron/hole dispersion over the whole Brillouin zone, (ii) the 7-band model [47] which includes three $d$ orbitals of Metal, two $p$-orbitals for each Chalcogen and only nearest neighbour interactions (this model involves as well overlap matrix elements since it is developed for non-orthogonalized orbitals); (iii) the 11-band model of Ref. [48] which accounts for five $d$-orbitals of Metal and three $p$-orbitals of each Chalcogen and applies the nearest neighbour approximation; (iv) the 27-band model of Ref. [49] which takes into account $s p^{3} d^{5}$ states for each atom and provides rather high accuracy.

The diagonalization of the tight-binding Hamiltonian provides the energy dispersion in the whole Brillouin zone. In the vicinity of $\boldsymbol{K}^{ \pm}$points the tight-binding Hamiltonian can be expanded in power series in the electron wavevector (referred to the Brillouin zone edge) and can be partially diagonalized. The resulting effective Hamiltonian has a form similar to the $\boldsymbol{k} \cdot \boldsymbol{p}$ Hamiltonian (with different number of bands depending on the tight-binding model) but contains diagonal $k^{2}$ contributions. It is convenient to interpret the latter as an effective $\boldsymbol{k} \cdot \boldsymbol{p}$ contribution of "the bare electron mass and remote bands". The off-diagonal elements (linear in $\boldsymbol{k}$ ) contain coefficients which can be interpreted as inter-center contributions to the momentum matrix elements [50-53]. For example, the 3 -band model of Ref. [35] yields a $3 \times 3$ effective Hamiltonian which includes the $c, v$ and $c+2$ bands in notations of the $\boldsymbol{k} \cdot \boldsymbol{p}$ Hamiltonian (8). The 7band model of Ref. [47] yields an additional conduction band with the same symmetry as the $c$-band.

Within the tight-binding approach the magnetic field is also included in a two-fold way. First, the phase of the hopping matrix elements is modified by including the vector potential as follows

$$
-\frac{\mathrm{i} e}{c \hbar} \int_{\boldsymbol{r}_{1}}^{\boldsymbol{r}_{2}} \boldsymbol{A}(\boldsymbol{r}) \mathrm{d} \boldsymbol{r} .
$$

This procedure is equivalent to the $\boldsymbol{k} \rightarrow \boldsymbol{k}-(e / c \hbar) \boldsymbol{A}$ replacement in the $\boldsymbol{k} \cdot \boldsymbol{p}$ Hamiltonian. Second, the intraatomic contribution should be included. It contains the spin part, Eq. (4), as well as possible orbital contribution. The latter should be carefully calculated using the restricted basis of atomic orbitals, the completeness relation for this restricted basis, and an analogue of Eq. (6) in the form

$$
\begin{aligned}
& \left\langle\phi_{j}^{a}(\boldsymbol{r})\left|\hbar L_{z}\right| \phi_{j}^{a}(\boldsymbol{r})\right\rangle \\
& =\sum_{i}\left(\left\langle\phi_{j}^{a}|\hat{x}| \phi_{i}^{a}\right\rangle\left\langle\phi_{i}^{a}\left|\hat{p}_{y}\right| \phi_{j}^{a}\right\rangle-\left\langle\phi_{j}^{a}|\hat{y}| \phi_{i}^{a}\right\rangle\left\langle\phi_{i}^{a}\left|\hat{p}_{x}\right| \phi_{j}^{a}\right\rangle\right) .
\end{aligned}
$$

Note, that the summation in Eq. (15) extends over the orbitals used in Eq. (13) rather than over all possible states of an isolated atom, otherwise the problem becomes illdefined.

In the particular cases of 3-, 7- and 11-band tightbinding models, the intra-site values of the momentum operator vanish due to symmetry reasons because 
for any given atom either only $p$-shell or $d$-shell states are included into the model. Therefore, in Eq. (15) $\left\langle\phi_{j}^{a}(\boldsymbol{r})\left|\hbar L_{z}\right| \phi_{j}^{a}(\boldsymbol{r})\right\rangle=0$ and the intra-site contribution to the Zeeman effect vanishes. In this case we obtain within the tight-binding method Eq. (7) for the orbital momentum and recover expressions analogous to Eq. (11), Eqs. (12) from $\boldsymbol{k} \cdot \boldsymbol{p}$ theory with no additional intracenter contributions in contrast to Refs. [22-24], see also Ref. [54]. Additional intra-site contributions may arise in the advanced tight-binding formalism of Ref. [49] where orbitals of different symmetry are included for a given atom, in which case the momentum operator matrix elements between these orbitals may become non-zero.

\section{Zeeman splittings of direct excitons and trions}

The neutral exciton radiative decay involving emission of $\sigma^{+}$or $\sigma^{-}$photons results from the recombination of a Coulomb-correlated electron-hole pair in the $\boldsymbol{K}^{+}$or $\boldsymbol{K}^{-}$ valley, respectively. The Zeeman splitting of $\mathrm{X}^{0}$ is, in accordance with Eq. (2), given by

$$
\Delta_{Z}=\frac{1}{2}\left[g_{c}^{\boldsymbol{K}_{+}}-g_{v}^{\boldsymbol{K}_{+}}-\left(g_{c}^{\boldsymbol{K}_{-}}-g_{v}^{\boldsymbol{K}_{-}}\right)\right] \mu_{B} B_{z} .
$$

Note that hereafter we neglect the renormalization of $g$-factor due to the Coulomb effects and band nonparabolicity. Making use of Eqs. (11), (12) the bright exciton $g$-factor is given by

$$
g_{x}=g_{c}-g_{v}=-2\left(\frac{m_{0}}{m_{c}^{\prime}}+\frac{m_{0}}{m_{v}^{\prime}}\right)+\Delta g_{c}^{\mathrm{orb}}-\Delta g_{v}^{\mathrm{orb}},
$$

where terms in parenthesis are calculated within the framework of Hamiltonian (4) and 4-band Hamitonian (8), and the contribution $\Delta g_{c}^{o r b}-\Delta g_{v}^{\text {orb }}$ results form the remote bands not accounted for by Eq. (8). At this stage precise measurements of the conduction band effective masses have not been reported yet. First measured values for the valence band effective masses can be obtained from angle-resolved photoemission spectroscopy (ARPES) [3, 55]. Equation (17) can also be represented in the form

$$
\begin{aligned}
g_{x}=4 & -2\left(\frac{m_{0}}{m_{c}}+\frac{m_{0}}{m_{v}}-\frac{m_{0}}{m_{c}^{\prime \prime}}-\frac{m_{0}}{m_{v}^{\prime \prime}}\right) \\
& +\Delta g_{c}^{\text {orb }}-\Delta g_{v}^{\text {orb }}
\end{aligned}
$$

where the contributions to the Landé factor and effective masses resulting from remote bands are explicitly present. Note that in the two-band approximation the bright exciton $g$-factor exactly vanishes because in this approximation the conduction- and valence-band electron effective masses are given by $m_{0} / m_{c}^{2 b}=1+m_{0} / m^{*}$, $m_{0} / m_{v}^{2 b}=1-m_{0} / m^{*}$ and, therefore,

$$
g_{x}^{2 b}=4-2\left(\frac{m_{0}}{m_{c}^{2 b}}+\frac{m_{0}}{m_{v}^{2 b}}\right)=4-2 \times 2=0 .
$$

The term 4 arises from inclusion of $1 / m_{0}$ in the inverse effective masses for the conduction and valence band electrons. It should be emphasized that the inclusion of free electron dispersion $1 / m_{0}$ in Eqs. (9) and neglecting the terms $1 / m_{c}^{\prime \prime}, 1 / m_{v}^{\prime \prime}, \Delta g_{c}^{o r b}$, and $\Delta g_{v}^{\text {orb }}$ contributed by remote bands cannot formally be justified within the $\boldsymbol{k} \cdot \boldsymbol{p}$ method. Those contributions should be estimated from experimental data or evaluated via more advanced $\boldsymbol{k} \cdot \boldsymbol{p}$ schemes or atomistic approaches.

Since in $\mathrm{MoSe}_{2}$ and $\mathrm{WSe}_{2}$ the conduction- and valenceband effective masses are close in absolute values but have opposite signs, in accordance with Eqs. (17), (18), the orbital contribution to the exciton $g$-factor stems from remote bands. We note that neither Eq. (17) with $\Delta g_{c}^{\text {orb }}-\Delta g_{v}^{\text {orb }}=0$ nor Eq. (18) with $m_{v}^{\prime \prime}, m_{c}^{\prime \prime} \rightarrow \infty$ can satisfactory describe the experiment in respect of both the sign and the magnitude of $g$-factor. Hence we conclude that the remote band contributions are important. For instance, if we add into consideration one more (distant) band with the same symmetry as the conduction band with band edge energy $E_{c}^{\prime}$ and the matrix element of $\boldsymbol{k} \cdot \boldsymbol{p}$ interaction with the valence band $\gamma_{3}^{\prime}$ we obtain an additional contribution to the valence band $g$-factor $\Delta g_{v}=4 m_{0} \gamma_{3}^{\prime 2} /\left[\hbar^{2}\left(E_{c}^{\prime}-E_{v}\right)\right]$, while $\Delta g_{c}=0$ for the symmetry reasons. Further experimental studies and theoretical modelling are therefore needed to elucidate the values of Zeeman splitting.

The Zeeman splitting for the bright trion is also given by Eq. (16). This is because the optical recombination involves charge carriers of opposite signs in the same valley, while the spin/valley state of the third carrier is not changed. The difference of measured values for excitons and trions can be attributed to the Coulombinduced renormalization of $g$-factors due to band nonparabolicity.

\section{E. Polarization of emission}

The polarization of the PL emission is governed by the selection rules and occupancies of spin/valley states of the carriers and Coulomb complexes. In case of $\mathrm{MoSe}_{2}$, neither optical orientation (circular polarization of photoluminescence for circularly polarized excitation at $\boldsymbol{B}=0$ ) nor exciton alignment or valley coherence (linear polarization at linearly polarized excitation) are observed [30]. This allows us to assume that in this material the spin/valley relaxation of both neutral excitons and trions (or individual carriers) is fast compared to the $\mathrm{PL}$ emission time. In the presence of a magnetic field the magneto-induced circular polarization seems to result from the preferential occupation of the lowest Zeeman state of the exciton or trion. It is indeed consistent with experiment where, for $B_{z}>0$, the state emitting the $\sigma^{+}$polarized photons has the lower energy and is dominant in the photoluminescence so that $P_{c}\left(B_{z}>0\right)>0$, see Fig. 1. Note, however, that the full thermalization to the lattice temperature does not occur because at $\left|B_{z}\right| \approx 9 \mathrm{~T}$ 
the Zeeman splitting exceeds by far the temperature expressed in the energy units, while experiment demonstrates only $\left|P_{c}\right| \sim 30 \%$. The effective spin temperature deduced from the experiment is about $T_{\mathrm{spin}}=30 \mathrm{~K}$.

The situation is more complex for $\mathrm{WSe}_{2}$ where the experiment shows a substantial optical orientation, Fig. 2f, and neutral-exciton alignment, Fig. 2e, even at $B_{z}=0$. In this case the spin/valley relaxation time is comparable to the lifetime of excitations and, in an applied magnetic field, thermalization may not occur. Additionally, as compared to $\mathrm{MoSe}_{2}, \mathrm{WSe}_{2}$ is characterized by the opposite sign of zero-field spin splitting of the conduction band [34, 35]. Hence, for the exciton and trion ground states the direct intra-valley optical transitions are spin-forbidden. These states can be manifested in optical spectra due to indirect (e.g. phonon-assisted) transitions similarly to the case of Carbon nanotubes [56]. It follows from the symmetry considerations that in this case the polarization is reversed [57]. Further experimental data using gated devices where positively and negatively charged excitons can be clearly distinguished and theoretical analysis are needed to clarify this issue.

Finally we emphasize that the above analysis is based on the perturbative treatment of the Coulomb interac- tion while, in TMDC MLs, the exciton binding energy amounts to $\gtrsim 0.5 \mathrm{eV}$ and, hence, is comparable to the band gap. Theoretical estimates show that depending on the parameters of the materials and dielectric environment the binding energy is so large that the $1 s$ state emission could be in the infra-red range and, moreover, the exciton ground state could even collapse $[58,59]$. In the former case the optical transition $2 p \rightarrow 1 s$ could be relevant as well, its Zeeman splitting and polarization deserve further study. In the latter case the ground state of the system could be strongly renormalized and an excitonic insulator could be formed [60-63]. Its polarization and magnetic field properties should also be studied in future works.

Acknowledgements. - We thank Andor Kormanyos, Hanan Dery, Iann Gerber and Junichiro Kono for very fruitful discussions. We acknowledge partial funding from ERC Grant No. 306719, ANR MoS2ValleyControl, Programme Investissements d'Avenir ANR-11-IDEX-0002-02, reference ANR-10LABX-0037-NEXT, RFBR, RF President grants MD5726.2015.2 and NSh-1085.2014.2 and Dynasty Foundation - ICFPM.
[1] K. F. Mak, C. Lee, J. Hone, J. Shan, and T. F. Heinz, Phys. Rev. Lett. 105, 136805 (2010).

[2] A. Splendiani, L. Sun, Y. Zhang, T. Li, J. Kim, C.-Y. Chim, G. Galli, and F. Wang, Nano Letters 10, 1271 (2010).

[3] Y. Zhang, T.-R. Chang, B. Zhou, Y.-T. Cui, H. Yan, Z. Liu, F. Schmitt, J. Lee, R. Moore, Y. Chen, et al., Nature Nanotechnology 9, 111 (2014).

[4] T. Cheiwchanchamnangij and W. R. L. Lambrecht, Phys. Rev. B 85, 205302 (2012).

[5] H.-P. Komsa and A. V. Krasheninnikov, Phys. Rev. B 86, 241201 (2012).

[6] J. S. Ross, S. Wu, H. Yu, N. J. Ghimire, A. M. Jones, G. Aivazian, J. Yan, D. G. Mandrus, D. Xiao, W. Yao, et al., Nature Communications 4, 1474 (2013).

[7] K. He, N. Kumar, L. Zhao, Z. Wang, K. F. Mak, H. Zhao, and J. Shan, Phys. Rev. Lett. 113, 026803 (2014).

[8] M. M. Ugeda, A. J. Bradley, S.-F. Shi, F. H. da Jornada, Y. Zhang, D. Y. Qiu, S.-K. Mo, Z. Hussain, Z.-X. Shen, F. Wang, et al., Nature Materials doi: 10.1038/nmat4061 (2014).

[9] A. Chernikov, T. C. Berkelbach, H. M. Hill, A. Rigosi, Y. Li, O. B. Aslan, D. R. Reichman, M. S. Hybertsen, and T. F. Heinz, Phys. Rev. Lett. 113, 076802 (2014).

[10] Z. Ye, T. Cao, K. O'Brien, H. Zhu, X. Yin, Y. Wang, S. G. Louie, and X. Zhang, Nature 513, 214 (2014).

[11] G. Wang, X. Marie, I. Gerber, T. Amand, D. Lagarde, L. Bouet, M. Vidal, A. Balocchi, and B. Urbaszek, Phys. Rev. Lett. 114, 097403 (2015).

[12] A. R. Klots, A. K. M. Newaz, B. Wang, D. Prasai, H. Krzyzanowska, D. Caudel, N. J. Ghimire, J. Yan, B. L. Ivanov, K. A. Velizhanin, et al., Scientific Reports 4, 6608 (2014).
[13] D. Xiao, G.-B. Liu, W. Feng, X. Xu, and W. Yao, Phys. Rev. Lett. 108, 196802 (2012).

[14] T. Cao, G. Wang, W. Han, H. Ye, C. Zhu, J. Shi, Q. Niu, P. Tan, E. Wang, B. Liu, et al., Nature Communications 3, 887 (2012).

[15] K. F. Mak, K. He, J. Shan, and T. F. Heinz, Nat. Nanotechnol. 7, 494 (2012).

[16] G. Sallen, L. Bouet, X. Marie, G. Wang, C. R. Zhu, W. P. Han, Y. Lu, P. H. Tan, T. Amand, B. L. Liu, et al., Phys. Rev. B 86, 081301 (2012).

[17] G. Kioseoglou, A. T. Hanbicki, M. Currie, A. L. Friedman, D. Gunlycke, and B. T. Jonker, Applied Physics Letters 101, 221907 (pages 4) (2012).

[18] A. M. Jones, H. Yu, N. J. Ghimire, S. Wu, G. Aivazian, J. S. Ross, B. Zhao, J. Yan, D. G. Mandrus, D. Xiao, et al., Nat. Nanotechnol. 8, 634 (2013).

[19] K. F. Mak, K. L. McGill, J. Park, and P. L. McEuen, Science 344, 1489 (2014).

[20] S. Najmaei, A. Mlayah, A. Arbouet, C. Girard, J. Leotin, and J. Lou, ACS Nano 8, 12682 (2014), pMID: 25469686.

[21] E. L. Ivchenko and G. Pikus, Superlattices and other Heterostructures, Symmetry and Optical Phenomena, Springer Series in Solid State Science 110, Springer Verlag Berlin Heildelberg (1995).

[22] D. MacNeill, C. Heikes, K. F. Mak, Z. Anderson, A. Kormányos, V. Zólyomi, J. Park, and D. C. Ralph, Phys. Rev. Lett. 114, 037401 (2015), URL http://link. aps.org/doi/10.1103/PhysRevLett.114.037401.

[23] Y. Li, J. Ludwig, T. Low, A. Chernikov, X. Cui, G. Arefe, Y. D. Kim, A. M. van der Zande, A. Rigosi, H. M. Hill, et al., Phys. Rev. Lett. 113, 266804 (2014), URL http: // link.aps.org/doi/10.1103/PhysRevLett.113.266804.

[24] G. Aivazian, Z. Gong, A. M. Jones, R.-L. Chu, J. Yan, 
D. G. Mandrus, C. Zhang, D. Cobden, W. Yao, and X. Xu, Nature Physics 11, 148 (2015).

[25] A. Srivastava, M. Sidler, A. V. Allain, D. S. Lembke, A. Kis, and A. Imamoglu, Nature Physics 11, 141 (2015).

[26] H. Zeng, J. Dai, W. Yao, D. Xiao, and X. Cui, Nat. Nanotechnol. 7, 490 (2012).

[27] A. Castellanos-Gomez, M. Buscema, R. Molenaar, V. Singh, L. Janssen, H. S. J. van der Zant, and G. A. Steele, 2D Materials 1, 011002 (2014), URL http:// stacks.iop.org/2053-1583/1/i=1/a=011002.

[28] L. Bouet, M. Vidal, T. Mano, N. Ha, T. Kuroda, M. V. Durnev, M. M. Glazov, E. L. Ivchenko, X. Marie, T. Amand, et al., Applied Physics Letters 105, 082111 (2014), URL http://scitation.aip.org/content/aip/ journal/apl/105/8/10.1063/1.4894174.

[29] B. Urbaszek, X. Marie, T. Amand, O. Krebs, P. Voisin, P. Maletinsky, A. Högele, and A. Imamoglu, Rev. Mod. Phys. 85, 79 (2013).

[30] G. Wang, E. Palleau, T. Amand, S. Tongay, X. Marie, and B. Urbaszek, ArXiv e-prints (2015), 1502.03591.

[31] G. Wang, L. Bouet, D. Lagarde, M. Vidal, A. Balocchi, T. Amand, X. Marie, and B. Urbaszek, Phys. Rev. B 90, 075413 (2014).

[32] H. Yu, G. Liu, P. Gong, X. Xu, and W. Yao, Nature Comms. 5, 3876 (2014).

[33] M. Dyakonov, Springer Series in Solid-State Science, Springer-Verlag Berlin 157 (2008).

[34] K. Kosmider, J. W. González, and J. Fernández-Rossier, Phys. Rev. B 88, 245436 (2013), URL http: //link. aps . org/doi/10.1103/PhysRevB.88.245436.

[35] G.-B. Liu, W.-Y. Shan, Y. Yao, W. Yao, and D. Xiao, Phys. Rev. B 88, 085433 (2013), URL http://link.aps. org/doi/10.1103/PhysRevB.88.085433.

[36] M. Z. Maialle, E. A. de Andrada e Silva, and L. J. Sham, Phys. Rev. B 47, 15776 (1993), URL http://link.aps. org/doi/10.1103/PhysRevB.47.15776.

[37] M. M. Glazov, T. Amand, X. Marie, D. Lagarde, L. Bouet, and B. Urbaszek, Phys. Rev. B 89, 201302 (2014).

[38] T. Yu and M. W. Wu, Phys. Rev. B 89, 205303 (2014).

[39] L. Landau and E. Lifshitz, Quantum Mechanics: NonRelativistic Theory (vol. 3) (Butterworth-Heinemann, Oxford, 1977).

[40] E. L. Ivchenko, Optical spectroscopy of semiconductor nanostructures (Alpha Science, Harrow UK, 2005).

[41] A. Kormányos, V. Zólyomi, N. D. Drummond, P. Rakyta, G. Burkard, and V. I. Fal'ko, Phys. Rev. B 88, 045416 (2013), URL http://link.aps.org/doi/10. 1103/PhysRevB.88.045416.

[42] A. Kormányos, V. Zólyomi, N. D. Drummond, and G. Burkard, Phys. Rev. X 4, 011034 (2014).

[43] A. Kormanyos, G. Burkard, M. Gmitra, J. Fabian, V. Zolyomi, N. D. Drummond, and V. Fal'ko, ArXiv eprints (2014), 1410.6666.

[44] G. L. Bir and G. E. Pikus, Symmetry and Strain-induced
Effects in Semiconductors (Wiley/Halsted Press, 1974).

[45] P. O. Löwdin, The Journal of Chemical Physics 18, 365 (1950), URL http://scitation.aip.org/content/aip/ journal/jcp/18/3/10.1063/1.1747632.

[46] G.-B. Liu, D. Xiao, Y. Yao, X. Xu, and W. Yao, Chem. Soc. Rev. pp. - (2015), URL http://dx.doi.org/10. 1039/C4CS00301B.

[47] H. Rostami, A. G. Moghaddam, and R. Asgari, Phys. Rev. B 88, 085440 (2013), URL http://link.aps.org/ doi/10.1103/PhysRevB.88.085440.

[48] E. Cappelluti, R. Roldán, J. A. Silva-Guillén, P. Ordejón, and F. Guinea, Phys. Rev. B 88, 075409 (2013), URL http://link.aps.org/doi/10. 1103/PhysRevB.88.075409.

[49] F. Zahid, L. Liu, Y. Zhu, J. Wang, and H. Guo, AIP Advances 3, 052111 (2013), URL http://scitation.aip.org/content/aip/journal/ adva/3/5/10.1063/1.4804936.

[50] L. C. Lew Yan Voon and L. R. Ram-Mohan, Phys. Rev. B 47, 15500 (1993), URL http://link.aps.org/doi/ 10.1103/PhysRevB.47.15500.

[51] M. Graf and P. Vogl, Phys. Rev. B 51, 4940 (1995), URL http://link.aps.org/doi/10.1103/PhysRevB. 51.4940 .

[52] S. Goupalov and E. Ivchenko, Physics of the Solid State 43, 1867 (2001).

[53] Z. Xu, Solid State Communications 76, 1143 (1990), ISSN 0038-1098, URL http://www.sciencedirect.com/ science/article/pii/003810989090981G.

[54] In the well-studied case of GaAs the inclusion of atomic value for Landé-factor for $J=3 / 2$ orbital yields valence band $g$-factor of $+4 / 3$ instead of known value of $g=-2.4$ resulting from isotropic Zeeman term $-2 \mu_{B} \varkappa(\boldsymbol{J} \cdot \boldsymbol{B})$ [21].

[55] J. M. Riley, F. Mazzola, M. Dendzik, M. Michiardi, T. Takayama, L. Bawden, C. Granerod, M. Leandersson, T. Balasubramanian, M. Hoesch, et al., Nat Phys 10, 835 (2014).

[56] A. Srivastava, H. Htoon, V. I. Klimov, and J. Kono, Phys. Rev. Lett. 101, 087402 (2008), URL http://link.aps. $\mathrm{org} /$ doi/10.1103/PhysRevLett.101.087402.

[57] M. M. Glazov and E. L. Ivchenko, to be published.

[58] A. S. Rodin and A. H. Castro Neto, Phys. Rev. B 88, 195437 (2013), URL http://link.aps.org/doi/10. 1103/PhysRevB.88.195437.

[59] T. Stroucken and S. W. Koch, ArXiv e-prints (2014), 1404.4238.

[60] L. Keldysh and Y. V. Kopaev, SOVIET PHYSICS SOLID STATE, USSR 6, 2219 (1965).

[61] A. N. Kozlov and L. A. Maksimov, JETP 21, 790 (1965).

[62] D. Jérome, T. M. Rice, and W. Kohn, Phys. Rev. 158, 462 (1967), URL http://link.aps.org/doi/10.1103/ PhysRev.158.462.

[63] R. R. Guseinov and L. V. Keldysh, JETP 36, 1193 (1973). 